\title{
Turning plants from passive to active material: FERONIA and microtubules independently contribute to mechanical feedback
}

Alice Malivert ${ }^{1}$, Özer Erguvan ${ }^{1,2}$, Antoine Chevallier ${ }^{1}$, Antoine Dehem ${ }^{1}$, Rodrigue Friaud ${ }^{1}$, Mengying Liu ${ }^{1}$, Marjolaine Martin ${ }^{1}$, Théophile Peyraud ${ }^{1}$, Olivier Hamant ${ }^{1, *}$, Stéphane Verger $^{1,2, *}$

* Correspondence: olivier.hamant@ens-lyon.fr $(\mathrm{OH})$, stephane.verger@slu.se (SV)

1. Laboratoire de Reproduction et Développement des Plantes, Université de Lyon, UCB Lyon 1, ENS de Lyon, INRAE, CNRS, 46 Allée d'Italie, 69364 Lyon Cedex 07, France

2. Present address : Umeå Plant Science Centre, Department of Forest Genetics and Plant Physiology, Swedish University of Agricultural Sciences, SE-901 83 Umeå, Sweden

\begin{abstract}
To survive, cells must constantly resist mechanical stress. In plants, this involves the reinforcement of cell walls, notably through microtubule-dependent cellulose deposition, and thus wall sensing. Several receptor-like kinases have been proposed to act as mechanosensors. Here we tested whether the microtubule response to stress acts downstream of known wall sensors. Using a multi-step screen with eleven mutant lines, we identify FERONIA as the primary candidate for controlling the microtubule response to stress. However, when performing mechanical perturbations, we show that the microtubule response to stress can be independent from FER. We reveal that the feronia phenotype can be partially rescued by reducing tensile stress levels. Conversely, in the absence of both microtubules and FER, cells swell and burst like soap bubbles. Altogether, this shows that the microtubule response to stress acts as an independent pathway to resist stress, in parallel to FER. We propose that both pathways are key components to turn plant cells from passive to active material.
\end{abstract}




\section{Introduction}

All living organisms use mechanical forces as instructive cues during their development (Wolfenson et al., 2019)(Hamant and Saunders, 2020). They also share a common mechanical property: cells are pressurized by osmotic pressure and thus experience cortical tension. Osmotic pressure in plants is several orders of magnitude higher than that of animal cells, and it is counterbalanced by stiff cell walls (Schopfer, 2006). Regulating the mechanical properties of cell walls, through the perception of wall tension and integrity, is thus crucial for plant growth and development (Bacete and Hamann, 2020)(Trinh et al., 2021).

Plant cell walls are composed of load-bearing cellulose microfibrils, tethered by a matrix made of polysaccharides and structural proteins (Cosgrove, 2005)(Anderson and Kieber, 2020). The deposition of cellulose microfibrils is generally guided by cortical microtubules (Paredez et al., 2006). Beyond the average stiffness, the orientation of cellulose microfibrils controls the mechanical anisotropy of the wall. There is now ample evidence showing that cortical microtubules align with maximal tensile stress directions (Green and King, 1966)(Williamson, 1990)(Hejnowicz et al., 2000)(Hamant et al., 2008)(Verger et al., 2018). This provides a feedback loop in which shape and growth, whether at the individual cell or whole organ scale, prescribes a pattern of stress, to which cells resist by reinforcing their walls along maximal tensile stress directions (Hamant et al., 2008)(Sampathkumar et al., 2014).

Although they are significantly less stiff, other wall components contribute to wall properties. In particular, pectins can partially rescue defects in cellulose synthesis in young cell walls. For instance, isoxaben treatment, which inhibits cellulose deposition through the internalization of CESA complexes, leads to thicker walls that are enriched in pectin (Manfield et al., 2004). Similarly, in young hypocotyls, pectin polarities precede the formation of mechanically anisotropic walls (Peaucelle et al., 2015). In contrast to cellulose deposition, pectin, as well as all other matrix components, are secreted to the cell wall (Cosgrove, 2005)(Anderson and Kieber, 2020). Therefore, in principle this provides an alternative mechanism for the cell to resist wall tension or damage. As for microtubules, the related mechanotransduction pathway is largely unknown. Yet, over the past decade, Catharanthus roseus Receptor-like kinases (CrRLKs) have emerged as key players. Although the link with mechanical stress remains to be formally established, THESEUS1 (THE1) has been involved in the wall integrity pathway (Hématy et al., 2007)(Gonneau et al., 2018)(Engelsdorf et al., 2018). Based on defective root growth behaviour on stiff interface, calcium signalling, $\mathrm{pH}$ response, and TOUCH gene 
expression, FERONIA (FER) acts as a mechanosensor (Shih et al., 2014). FER can sense the status of the cell wall, notably when salinity rises, through pectin binding (Feng et al., 2018). Last, it was recently proposed that FER also monitors microtubule behaviour through a cascade involving Rho GTPases (ROP6) and the microtubule severing protein katanin (Lin et al., 2018). Here, through a reverse genetic screen on wall sensors and using a suite of mechanical tests, we show that our best wall sensor candidate FER is not required for the microtubule response to stress, further suggesting that the microtubule response to stress can be more autonomous than anticipated. We also reveal that FER-dependent wall integrity pathway depends on wall tension, and that both FER and the microtubule response to stress contribute to wall integrity.

\section{Results}

\section{Altered pavement cell shape as a proxy for defective response to mechanical stress}

We first used a morphometric proxy to test the involvement of wall sensors in the microtubule response to stress. The shape of Arabidopsis pavement cells has been proposed to be actively maintained and amplified by the microtubule response to mechanical stress. Indeed, necks in such cells prescribe highly anisotropic tensile stresses locally, to which microtubule arrays and thus cellulose deposition align (Sampathkumar et al., 2014)(Sapala et al., 2018)(Bidhendi et al., 2019). We reasoned that the shape of pavement cells could be used in a mutant screen as a proxy for defects in that mechanical feedback loop. So far, such screens have been focused on either the intracellular biochemical cues behind cell-cell coordination (Fu et al., 2005) or the contribution of cell wall properties in cell shape (Majda et al., 2017). Whether wall sensors are involved in pavement cell shape remains ill-described. Here we focused on mutants impaired in receptor-like kinases that are highly expressed in the epidermis and aerial parts of the plant during early development and that exhibit an established link with the cell wall (and their closest homologs), namely feronia (fer), theseus 1 (the1), theseus 1/feronia-related1 (tfr1; at5g24010), curvyl (cvy1), hercules receptor kinase 1 (herk1), herk2, mdis1-interacting receptor-like kinase2 (mik2-1), wall-associated kinase 1 (wak1), wak2, wak3, wak4 (see Supplementary Table 1). We imaged and quantified the pavement cell shapes in receptor-like kinase candidate mutants, with the aim to select the ones with the strongest cell shape defects (Figure 1A). 
To do so, we extracted the epidermal signal and used PaCeQuant to segment pavement cells and measure 27 shape descriptors (see Material and methods, (Möller et al., 2017) (Supplementary Figure 1A)). To select the most discriminating PaCeQuant shape descriptor, we first performed a principal component analysis on our data. We compared the contribution of each parameter to the two axes with the most variation (Figure 1B). The cell perimeter was first, followed by the convex hull perimeter, the convex hull area, the cell area, the non-lobe area and the longest path length, then by circularity (for relevant definition, see Supplementary Figure 1B).

To confirm that such shape descriptors are pertinent, and knowing that cortical microtubules are well-known pavement cell shape regulators, we used mutants with microtubule defects as positive controls. We performed the same analysis on mutants with a reported enhanced microtubule response to stress (nek6 (Takatani et al., 2020)) and a reported reduced response to stress (bot1 (Uyttewaal et al., 2012). We also included lines with tubulin mutations affecting microtubule dynamics $\left(\right.$ tua $^{\text {D205N }}$, tua $^{\text {S1784 }}$, and tua $6^{A 281 T}$ referred as tua3, tua4 and tua 5 in the following (Ishida et al., 2007)(Matsumoto et al., 2010)) and spr2 with a reported enhanced cortical microtubule response to stress (Hervieux et al., 2016) but also ambivalent regulatory role in microtubule severing depending on tissue (Wightman et al., 2013)(Fan et al., 2018)(Nakamura et al., 2018). We found that cell perimeter, convex hull perimeter, convex hull area, cell area, and the longest path length descriptors were not sufficient to discriminate the pavement cell shape phenotype of those microtubules regulators (cell perimeter: $p_{\text {spr-2-2 }}=0.07$; $p_{\text {tua } 3}=0.018 ; p_{\text {tua }}=0.4$; convex hull perimeter: $p_{\text {tua } 3}=0.39 ; p_{\text {tua }}=0.011$; convex hull area: $p_{\text {tua } 3}=0.5 ; p_{\text {tua }}=0.03$; cell area: $p_{\text {tua } 3}=0.06 ; p_{\text {tua }}=0.07$; longest path length: $p_{\text {tua }}=0.28 ; p_{\text {tua }}=0.1$ $\left.\mathrm{n}_{\text {spr } 2-2}=262 ; \mathrm{n}_{\text {tua } 3}=204 ; \mathrm{n}_{\text {tua }}=230 ; \mathrm{n}_{\text {tua }}=297 ; \mathrm{n}_{\text {Col- }-0}=428\right)$. In contrast, all microtubule regulator mutant lines tested exhibited a defect in non-lobe area and circularity (non-lobe area: $p_{n e k 6-}$ ${ }_{I}=0.009 ; p_{\text {spr } 2-2}<10^{-3} ; p_{\text {tua } 3}=0.003 ; p_{\text {tua } 4}<10^{-3} ; p_{\text {tua } 5}<10^{-3} ; p_{\text {bot } 1-7}<10^{-3}$; circularity: $p_{\text {nek6-1 }}<10^{-3}$; $p_{\text {spr } 2-2}<10^{-3} ; p_{\text {tua } 3}<10^{-3} ; p_{\text {tua } 4}<10^{-3} ; p_{\text {tua } 5}<10^{-3} ; p_{\text {bot } 1-7}<10^{-3}, \mathrm{n}_{\text {nek } 6-1}=183 ; \mathrm{n}_{\text {spr } 2-2}=262 ; \mathrm{n}_{\text {tua } 3}=204$; $\mathrm{n}_{\text {tua }}=230 ; \mathrm{n}_{\text {tua }}=297 ; \mathrm{n}_{\text {bot } 1-7}=252 ; \mathrm{n}_{\text {Col- } 0}=428 ; \mathrm{n}_{\text {ws-4 }}=294$, Figure $1 \mathrm{C}$ and Supplementary Figure $1 \mathrm{C})$.

9 of the 11 receptor-like kinase mutants exhibited a non-lobe area significantly different than that of the WT. tfr1-1, fer-4, herk1-1, herk2-1, and wak4-1 displayed a non-lobe area significantly higher than that of the WT $\left(p_{\text {tfrl } 1-1}<10^{-3} ; p_{\text {fer }-4}<10^{-3} ; p_{\text {herk } 1-1}<10^{-3} ; p_{\text {herk } 2-1}<10^{-3} ; p_{\text {wak4- }}\right.$ ${ }_{I}=0.007 ; \quad \mathrm{n}_{\text {tfrl }-1}=244 ; \quad \mathrm{n}_{\text {fer }-4}=321 ; \quad \mathrm{n}_{\text {herk } 1-1}=225 ; \quad \mathrm{n}_{\text {herk } 2-1}=238 ; \quad \mathrm{n}_{\text {wak }-1}=204 ; \quad \mathrm{n}_{\text {Col }-0}=428$, Supplementary Figure 1C) while the non-lobe area of cvy1-1, the1-6, wakl-1 and mik2-1 was 
significantly lower than that of the WT $\left(p_{\text {cvyl-1 }}<10^{-3} ; p_{\text {thel- }}<10^{-3} ; p_{\text {wakl }-1}<10^{-3} ; p_{\text {mik2 }-1}<10^{-3} ; \mathrm{n}_{\text {cvyl- }}\right.$ $\left.{ }_{l}=277 ; \mathrm{n}_{\text {thel- } 6}=174 ; \mathrm{n}_{\text {wakl-1 }}=295 ; \mathrm{n}_{\text {mik2-1 }}=177 ; \mathrm{n}_{\text {Col- }-0}=428\right)$. Only wak2-1 and wak3-1 displayed non-lobe area values that were non-significantly different from that of the WT $\left(p_{\text {wak } 2-1}=0.1\right.$; $p_{\text {wak3-1 }}=0.03 ; \mathrm{n}_{\text {wak }-1}=271 ; \mathrm{n}_{\text {wak3-1 }}=202 ; \mathrm{n}_{\text {Col- }-0}=428$ ). Thus, non-lobe area is not a discriminant parameter in our screen. We decided to study the next most variable parameter with defects in known microtubule regulator lines - circularity - for the rest of the analysis, justifying $a$ posteriori a common choice in the literature on pavement cell shape (Zhang et al., 2011).

Among the receptor-like kinase mutant tested, the pavement cells in fer-4, the1-6, wak2-1 and mik2-1 were significantly more circular than the WT supporting the hypothesis that the corresponding proteins could contribute to the microtubule response to stress in pavement cells $\left(p_{\text {fer }-4}<10^{-3} ; p_{\text {thel-6 }}<10^{-3} ; p_{\text {wak2-1 }}=0.002 ; p_{\text {mik2-1 }}<10^{-3} ; \mathrm{n}_{\text {fer }-4}=321 ; \mathrm{n}_{\text {thel- }}=174 ; \mathrm{n}_{\text {wak } 2-1}=271 ; \mathrm{n}_{\text {mik2 }}\right.$ ${ }_{l}=177 ; \mathrm{n}_{\text {Col- }-0}=428$, Figure 1C). wakl-1 and tf $1-1$ also exhibited increased pavement cell circularity, albeit much less significantly $\left(p_{\text {wakl }-1}=0.04 ; p_{\text {tfrl }-1}=0.03 ; \mathrm{n}_{\text {wakl-l }}=295 ; \mathrm{n}_{\text {tfrl }-1}=244\right)$. Pavement cells in all the other receptor-like kinase mutants (cvy1-1, herk1-1; herk2-1; wak3-1; wak4-1) displayed a circularity comparable to that of the WT $\left(\mathrm{p}_{\text {cvyl-1 }}=0.49 ; p_{\text {herk } 1-1}=0.2 ; p_{\text {herk2- }}\right.$ ${ }_{I}=0.47 ; p_{\text {wak } 3-1}=0.47 ; p_{\text {wak } 4-1}=0.5 ; \mathrm{n}_{\text {cvyl-I }}=277 ; \mathrm{n}_{\text {herk } 1-1}=225 ; \mathrm{n}_{\text {herk } 2-1}=238 ; \mathrm{n}_{\text {wak3-I }}=202 ; \mathrm{n}_{\text {wak4- }}$ ${ }_{I}=204 ; \mathrm{n}_{\text {Col- }-0}=428$ ). To distinguish the relative contributions of the most affected mutants, we quantified the deviation of circularity from the WT. Among all the receptor-like kinases tested, the candidate mutants with the largest defect in circularity when compared to the WT were fer4 and, to a lesser extent, the 1-6 (Figure 1D). fer-4 cells were $30 \%$ more circular and the 1-6 cells were $7 \%$ more circular than the WT, values that were comparable to that of microtubule regulator mutants, such as spr2-2 or bot1-7 (Figure 1D).

Note that the same trend was obtained when considering solidity, another parameter often used to characterize lobe formation (Vőfély et al., 2019) (Supplementary Figure 1D). Altogether, these data suggest that fer-4 may have the most significant defect in microtubule dynamics hinting to a potential role of FERONIA in the microtubule response to mechanical stress. 

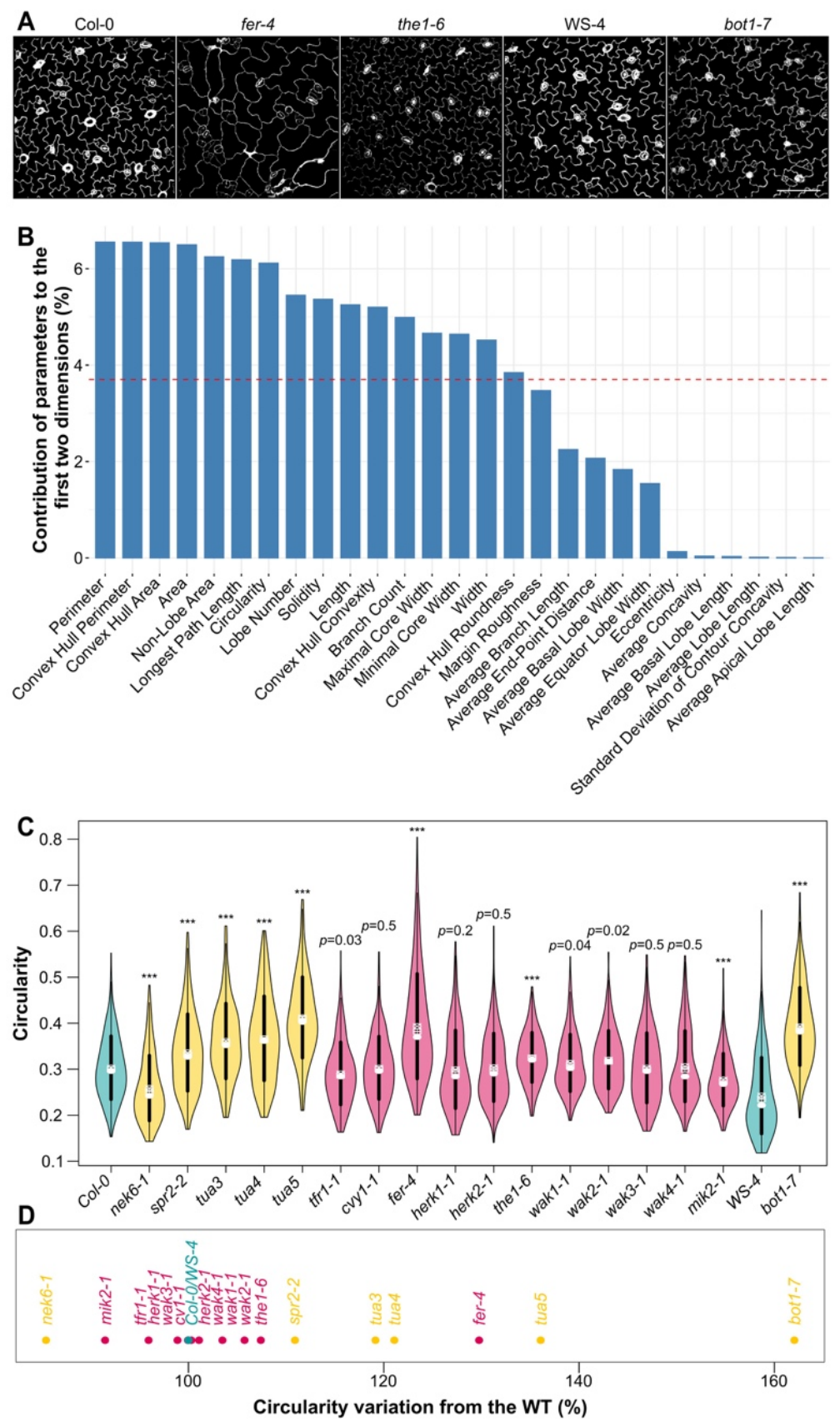

Figure 1. Pavement cell shape as a proxy for microtubule response to mechanical stress in receptor-like kinase mutants

A. Representative images of Col-0, WS-4, fer-4, the1-6 and bot1-7 pavement cells. Samples were PI stained and cell contours were extracted with MorphoGraphX and projected in 2D. Scale $=100 \mu \mathrm{m}$. B. Relative contribution of 27 shape descriptors to pavement cell shape, as assessed by Principal Component Analysis. C. Circularity (violin plots) of pavement cells and $p$-values ( $p$ ) of Dunn tests for the WT (in blue, Col-0, WS-4), for the microtubule regulator (in orange, nek6-1, spr2-2, tua3, tua4, tua5, bot1-7) and the receptor-like kinase mutants (in pink, tfr1-1, cvy1-1, fer-4, herk1-1, herk2-1, the 14, the1-6, wak1-1, wak2-1, wak3-1, wak4-1, mik2-1). D. Percentage of increase or decrease in pavement cell circularity from the WT (in blue, Col-0, WS-4), for the microtubule regulator (in orange, nek6-1, spr2-2, tua3, tua4, tua5, bot1-7) and the receptor-like kinase mutants (in pink, tfrl-1, cvyl-1, fer-4, herk1-1, herk2-1, the1-4, the1-6, wak1-1, wak2-1, wak3-1, wak4-1, mik2-1) 


\section{Differential response of receptor-like kinase mutants to isoxaben}

To challenge the results from this initial screen, we next used a well-established protocol to mechanically perturb cell walls. Isoxaben inhibits the biosynthesis of cellulose (Scheible et al., 2001), and thus weakens the wall. In past work, such treatment were shown to induce a hyperalignment of cortical microtubules at the shoot apical meristem and in cotyledon pavement cells (Heisler et al., 2010)(Sampathkumar et al., 2014), consistent with a response to increased tensile stress levels in the cell wall. Note that isoxaben can also trigger other responses, including ROS production, lignification, and changes in gene expression (Hématy et al., 2007). Thus, depending on time and dose, isoxaben may also ultimately reduce stress level (Engelsdorf et al., 2019).

We grew the seedlings in a medium containing $1 \mathrm{nM}$ isoxaben or the same volume of DMSO, in the dark (Figure 2A). We then measured the length of the etiolated hypocotyls four days after germination. After isoxaben treatment, four-day old WT seedlings exhibited a shorter hypocotyl (by $42 \%$ for Col-0, 34\% for WS-4, $\mathrm{n}_{\text {Col- } 0 \text { DMSO }}=287, \mathrm{n}_{\text {Col- } 0 \text { iso }}=271$, $\mathrm{n}_{\text {WS }-4 \text { DMSO }}=101, \mathrm{n}_{\text {WS-4 iso }}=96$ ). To compare WT and mutants, we normalized the obtained distribution of lengths to the same mean and standard deviation as the control, thus providing a hypocotyl length index (Figure 2B, Supplementary Figure 2A). Treated mutants with a relatively shorter hypocotyl than the treated WT were labelled more sensitive to isoxaben whereas treated mutants with a relatively longer hypocotyl than the treated WT were labelled less sensitive than the WT.

10 out of the 11 receptor-like kinase mutants studied were less sensitive than the WT (tfrl-1, cvy1-1, herk1-1, herk2-1, the1-6, wak1-1, wak2-1, wak3-1, wak4-1, mik2-1; $p_{\text {tfrl-1 }}<10^{-3}$; $p_{\text {cvyl- }}$ ${ }_{1}<10^{-3}$; pherk $1-1_{1}<10^{-3}$; pherk $2-1_{1}<10^{-3} ; p_{\text {thel- }-6}<10^{-3} ; p_{\text {wakl }-1}<10^{-3} ; p_{\text {wak } 2-1}<10^{-3} ; p_{\text {wak } 3-1}<10^{-3} ; p_{\text {wak3-1 }}<10^{-}$

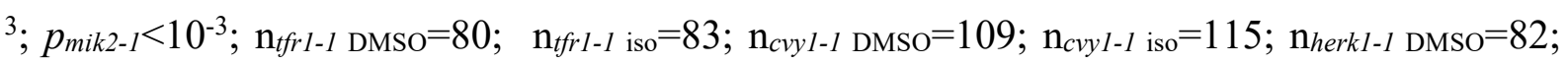
$\mathrm{n}_{\text {herk } 1-1 \text { iso }}=70 ; \mathrm{n}_{\text {herk } 2-1 \text { iso }}=88 ; \mathrm{n}_{\text {herk } 2-1 \text { iso }}=106 ; \mathrm{n}_{\text {thel- } 6 \text { DMSO }}=57 ; \mathrm{n}_{\text {thel- } 6 \text { iso }}=91 ; \mathrm{n}_{\text {wakl- } 1 \text { DMSO }}=116 ; \mathrm{n}_{\text {wakl- }}$ 1 iso $=89 ; \mathrm{n}_{\text {wak } 2-1 \text { DMSO }}=63 ; \mathrm{n}_{\text {wak } 2-1 \text { iso }}=78 ; \mathrm{n}_{\text {wak } 3-1 \text { DMSO }}=99 ; \mathrm{n}_{\text {wak } 3-1 \text { iso }}=89 ; \mathrm{n}_{\text {wak } 4-1 \text { DMSO }}=103 ; \mathrm{n}_{\text {wak4- } 1}$ iso $=86 ; \mathrm{n}_{\text {mik2-1 }}$ DMSO $=84 ; \mathrm{n}_{\text {mik2-1 }}$ iso $=96 ; \mathrm{n}_{\text {Col-0 DMSO }}=287 ; \mathrm{n}_{\text {Col- }-0}$ iso $=271$ ), whereas fer -4 was

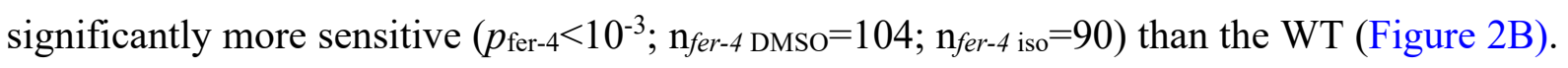
When plotting the deviation of each mutant from the WT phenotype, it appeared that among all the receptor-like kinase tested, fer-4 and the 1-6 were the most affected mutants in their response to isoxaben, albeit in opposite trend: fer-4 hypocotyl were more sensitive to isoxaben whereas the 1-6 were less sensitive to isoxaben (Figure 2C). These results indicate that all the receptorlike kinases tested might be involved in the cellular response to a mechanical stress, with FER and THE1 having the most clear-cut, and opposing, response. 

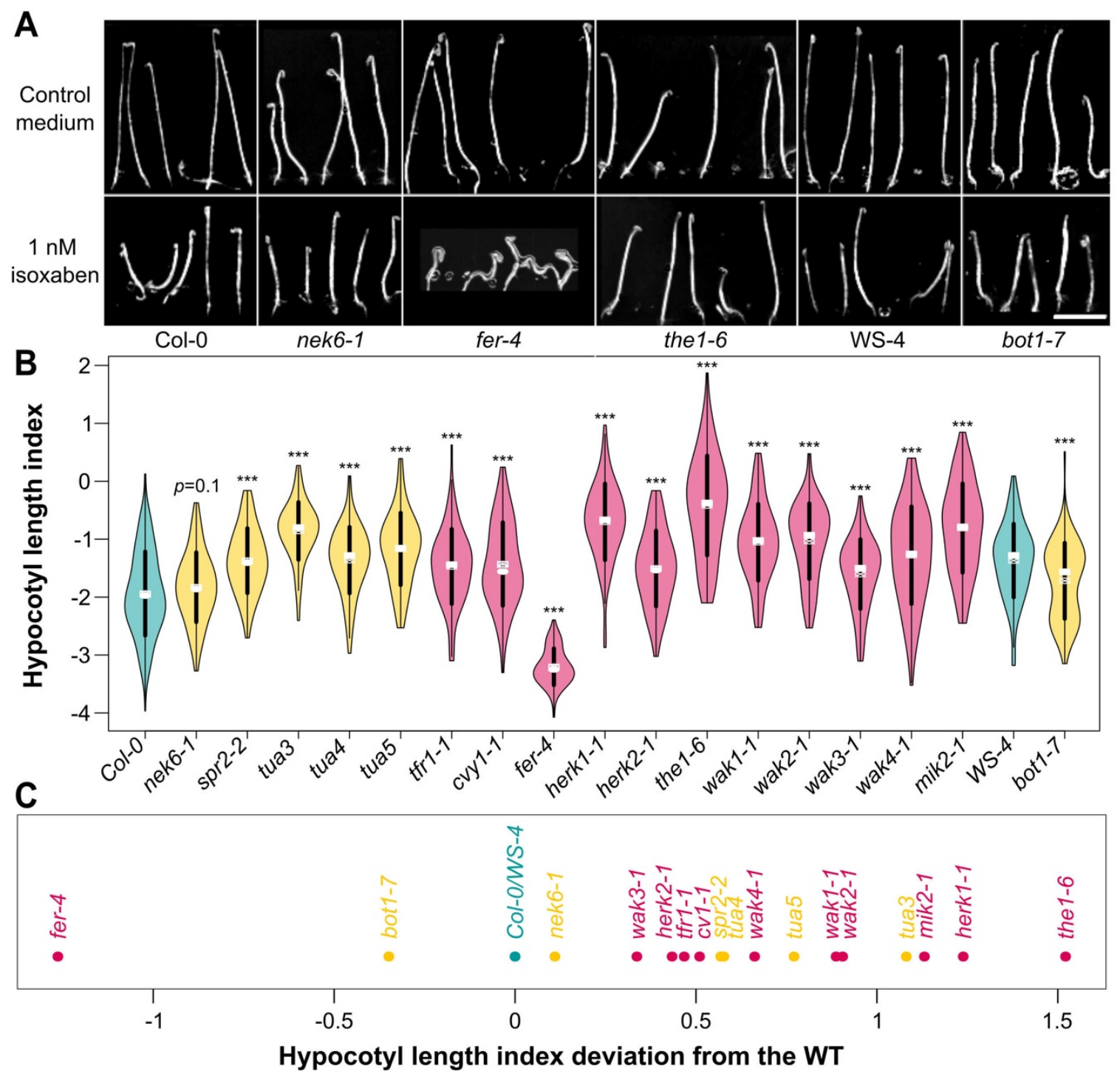

Figure 2. The impact of isoxaben on hypocotyl length reveals a differential contribution of receptorlike kinases in growth.

A. Representative images of Col-0, WS-4, fer-4, the 1-6 and bot1-7 etiolated seedlings grown with or without $1 \mathrm{nM}$ isoxaben. Scale=1 cm. B. Hypocotyl length index (violin plot): distribution of isoxabengrown hypocotyl length, normalized relative to the DMSO-grown ones. $p$-values $(p)$ of WilcoxonMann-Whitney test for the WT (in blue, Col-0, WS-4), the microtubule regulator (in orange, nek6-1, spr2-2, tua3, tua4, tua5, bot1-7), and the receptor-like kinase mutants (in pink, tfrl-1, cvyl-1, fer-4, herk1-1, herk2-1, the1-4, the1-6, wak1-1, wak2-1, wak3-1, wak4-1, mik2-1). C. Deviation of hypocotyl length index. The WT accessions (Col-0 and WS-4) are labelled in blue. The microtubule regulator mutant lines (nek6-1, spr2-2, tua3, tua4, tua5, bot1-7) are labelled in orange. The receptor-like kinase mutants (tfr1-1, cvy1-1, fer-4, herk1-1, herk2-1, the1-4, the1-6, wak1-1, wak2-1, wak3-1, wak4-1, mik21) are labelled in pink. 
To check whether these defects could be related to the microtubule response to stress, we performed the same analysis on microtubule regulator mutants. bot 1-7 (in WS-4 ecotype) was more sensitive to the isoxaben treatment than the WT $\left(p_{\text {bot }-7}<10^{-3} ; \mathrm{n}_{\text {botl }-7 \text { DMSO }}=99 ; \mathrm{n}_{\text {bot } 1-7 \text { iso }}=96\right.$; $\mathrm{n}_{\mathrm{WS}-4} \mathrm{DMSO}=101 ; \mathrm{n}_{\mathrm{WS}-4}$ iso $\left.=96\right)$ and thus fell in the same cluster as fer-4. The nek6-1 mutant exhibited the same isoxaben sensitivity as the WT $\left(p_{\text {nek6-l }}=0.15 ; \mathrm{n}_{\text {nek6-l }} \mathrm{DMSO}=91 ; \mathrm{n}_{\text {nek6- } 1 \text { iso }}=104\right.$; $\mathrm{n}_{\text {Col-0 DMSO }}=287 ; \mathrm{n}_{\text {Col-0 }}$ iso $\left.=271\right)$ (Figure $2 \mathrm{~B}$ ). The spr2-2 mutant was significantly less sensitive than the WT $\left(\mathrm{p}_{s p r 2-2}<10^{-3} ; \mathrm{n}_{s p r 2-2} \mathrm{DMSO}=99 ; \mathrm{n}_{s p r 2-2 \text { iso }}=85 ; \mathrm{n}_{\text {Col- } 0 \text { DMSO }}=287 ; \mathrm{n}_{\text {Col- } 0 \text { iso }}=271\right)$ and thus fell in the same cluster as the1-6. Last, tua3, tua 4 and tua 5 were significantly less sensitive than the WT $\left(p_{\text {tua } 3}<10^{-3} ; p_{\text {tua } 4}<10^{-3} ; p_{\text {tua } 5}<10^{-3} ; \mathrm{n}_{\text {tua } 3 \mathrm{DMSO}}=93 ; \mathrm{n}_{\text {tua } 3 \text { iso }}=90 ; \mathrm{n}_{\text {tua } 4 \text { DMSO }}=83 ; \mathrm{n}_{\text {tua } 4 \text { iso }}=108\right.$; $\mathrm{n}_{\text {tua } 5 \text { DMSO }}=72 ; \mathrm{n}_{\text {tua } 5 \text { iso }}=66 ; \mathrm{n}_{\text {Col- } 0 \text { DMSO }}=287 ; \mathrm{n}_{\text {Col- } 0 \text { iso }}=271$ ).

Altogether, both pavement cell shape analysis and isoxaben test single FER out. Our data are also consistent with a scenario in which FER and katanin could belong to the same pathway. Yet, the isoxaben test reveals that the relation between wall defects and cortical microtubule response is not simple. In the following we decided to focus on FER and disentangle these apparent contradictions.

\section{Rescue test: Decreasing growth medium matrix potential rescues the burst cell phenotype in fer-4}

It is commonly believed that WT seedlings display a shorter hypocotyl on isoxaben because cell wall defects are perceived and compensated through wall reinforcement, ultimately leading to reduced growth. Both wall reinforcement and reduced growth would prevent the cells from bursting. This notably builds on the analysis of the 1 mutant, which exhibits a longer hypocotyl than the WT on isoxaben because of the lack of wall sensing (Hématy et al., 2007). In fer, the hypocotyl is in contrast even shorter than the WT. Thus, either wall sensing is enhanced in fer, thus strongly repressing growth, or, in contrast, wall sensing is impaired in fer, even more than in the1, and cells burst before walls can even be reinforced. When looking closely at fer-4 hypocotyls grown on isoxaben and stained with propidium iodide (PI), we observed many dead cells, as revealed by PI staining (Figure 3A). We calculated a bursting index, i.e. the percentage of burst cell area over total area of a field of epidermal cells in a given image and found it to be much higher in fer-4 isoxaben-grown hypocotyls than in the WT $\left(p<10^{-3} ; \mathrm{n}_{\text {Col- }}\right.$ 0 ,isoxaben, $0.7 \%$ agar $=16 ; \mathrm{n}_{f e r-4 \text {,isoxaben, } 0.7 \% \text { agar }}=16$, Figure $3 \mathrm{~B}$ ). This observation thus seems consistent with the latter scenario. 
To test this hypothesis further, we reasoned that lowering the water potential in the medium should reduce water intake for seedlings, reduce tensile stress level (Verger et al., 2018), and in the end, reduce the number of burst cells. We thus tested different agar concentration in the medium and analysed the impact on fer-4 phenotype. When increasing the agar concentration to $2.5 \%$, the bursting index in hypocotyls was reduced by $70 \%$ in isoxaben-grown seedlings, supporting our hypothesis $\left(\mathrm{p}=0.002 ; \mathrm{n}_{\text {fer- }-4 \text {,isoxaben, } 0.7 \% \text { agar }}=16 ; \mathrm{n}_{\text {fer- }-4 \text {,isoxaben, } 2.5 \% \text { agar }}=16\right.$, Figure $3 \mathrm{~A}$ and 3B).

We then took a closer look at the pavement cells of fer-4 cotyledons grown in a medium containing a standard concentration of agar $(0.7 \%)$ and over time. Many burst cells were present here as well, as marked by a strong PI coloration (Figure 3C). The bursting index increased over time, from $16 \pm 16 \%$ at $4 \mathrm{DAG}$ (days after germination) to $60 \pm 30 \%$ at $12 \mathrm{DAG}$. At both time points, it was significantly superior to that of the WT ( $p_{4 \mathrm{DAG}, 0.7 \% \text { agar }}<10^{-3}$; $p_{12 \mathrm{DAG}, 0.7 \% \text { agar }}<10^{-3} ; \mathrm{n}_{\text {Col-0,4DAG, } 0.7 \% \mathrm{agar}}=14, \quad \mathrm{n}_{\text {Col-0,12DAG,0.7\%agar }}=9, \mathrm{n}_{\text {fer-4,4DAG, } 0.7 \% \text { agar }}=14, \mathrm{n}_{\text {fer }}$ 4,12DAG,0.7\%agar 8 ; Figure 3D). When we increased the agar concentration to $2.5 \%$, the bursting index in fer- 4 was still superior to that of the WT $\left(p_{4 \mathrm{DAG}, 2.5 \% \text { agar }}<10^{-3} ; p_{12 \mathrm{DAG}, 2.5 \% \text { agar }}=0.063 ; \mathrm{n}_{\mathrm{Col}}\right.$ $0,4 \mathrm{DAG}, 2.5 \% \mathrm{agar}=15, \mathrm{n}_{\text {Col-0,12DAG,2.5\%agar }} 9, \mathrm{n}_{\text {fer- }-4,4 \mathrm{DAG}, 2.5 \% \mathrm{agar}}=15, \mathrm{n}_{\text {fer }-4,12 \mathrm{DAG}, 2.5 \% \mathrm{agar}}=7$; Figure 3D). However, the bursting index was dramatically reduced to $1 \pm 4 \%$ at 4 DAG $\left(p_{f e r-4,4 \mathrm{DAG}}<10^{-3}\right)$ and to $14 \pm 19 \%$ at $12 \mathrm{DAG}\left(p_{f e r-4,12 \mathrm{DAG}}=0.003\right.$, Figure $\left.3 \mathrm{D}\right)$. Once again, lowering the water availability for cells partially rescued the bursting cell phenotype in fer-4.

To check whether the apparent rescue could have a more global effect on organ shape, we measured fer-4 cotyledon area over time on seedlings grown on media containing different agar concentrations $(0.7 \%, 2.5 \%$, Figure $3 \mathrm{E}$ and $3 \mathrm{~F})$. At a young stage (four days of light), fer-4

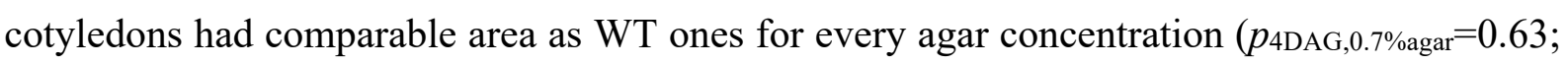
$p_{4 \mathrm{DAG}, 2.5 \% \mathrm{agar}}=0.44 ; \quad \mathrm{n}_{\text {Col-0,4DAG, } 0.7 \% \mathrm{agar}}=38 ; \quad \mathrm{n}_{\text {fer-4,4DAG, } 0.7 \% \mathrm{agar}}=23 ; \mathrm{n}_{\text {Col- } 0,4 \mathrm{DAG}, 2.5 \% \mathrm{agar}}=35 ; \quad \mathrm{n}_{\text {fer }}$ $4,4 \mathrm{DAG}, 2.5 \% \mathrm{agar}=27$, Figure $3 \mathrm{E}$ and $3 \mathrm{~F}$ ). After eight days of light, fer-4 cotyledons were $35 \%$ smaller than the WT for seedling grown on a medium containing $0.7 \%$ agar $\left(p_{8 \mathrm{DAG}, 0.7 \% \mathrm{agar}}<10^{-}\right.$ 3; $\left.\mathrm{n}_{\mathrm{Col}-0,8 \mathrm{DAG}, 0.7 \% \mathrm{agar}}=45 ; \mathrm{n}_{\text {fer }-4,8 \mathrm{DAG}, 0.7 \% \mathrm{agar}}=56\right)$. Strikingly, fer-4 cotyledons reached the same size as the WT for seedlings grown on $2.5 \%$ agar $\left(p_{8 \mathrm{DAG}, 2.5 \% \mathrm{agar}}=0.51 ; \mathrm{n}_{\mathrm{Col}-0,8 \mathrm{DAG}, 2.5 \% \mathrm{agar}}=55 ; \mathrm{n}_{\text {fer }}\right.$ $4,8 \mathrm{DAG}, 2.5 \% \mathrm{agar}=40$ ). However, this trend was not maintained after twelve days of light (Figure $3 \mathrm{E}$ and $3 \mathrm{~F})$.

Thus, we find a correlation between the mechanical status of the fer-4 cotyledons (as monitored by the frequency of cell burst) and growth. Taken together, these results suggest that the fer-4 mutant is unable to sense the mechanical status of the tissue, and that the fer- 4 phenotype 
reflects a passive turgor-dependent wall defect. In other words, the fer-4 mutation turns the plant from an active to a more passive material, where increased tensile stress leads to wall failure without feedback.
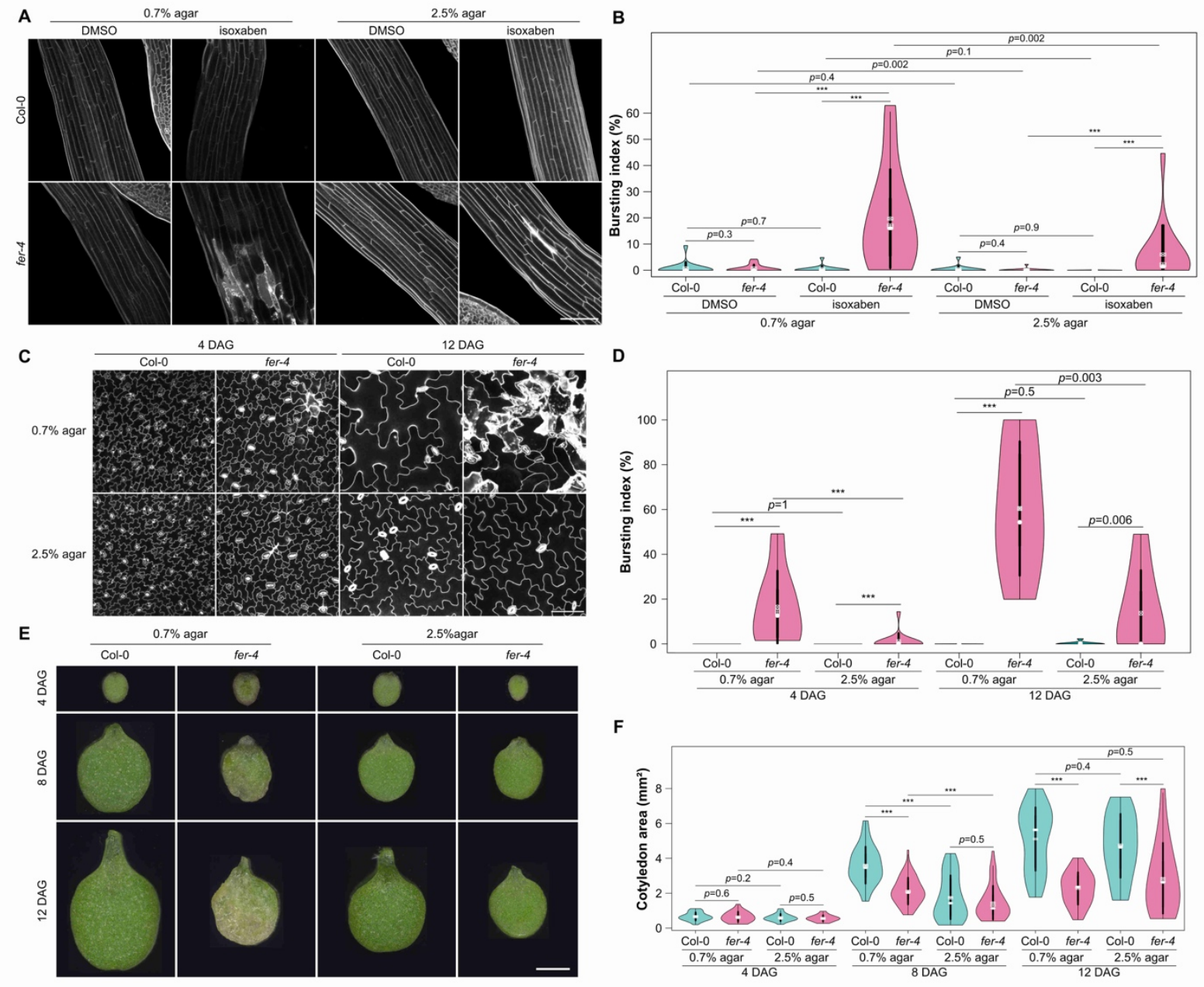

Figure 3. The fer phenotype can be partially rescued on $2.5 \%$ agar

A. Representative confocal images of Col-0 and fer-4 etiolated hypocotyls, from seedlings grown on a medium containing $1 \mathrm{nM}$ isoxaben with $0.7 \%$ or $2.5 \%$ agar (propidium iodide staining). Scale $=100 \mu \mathrm{m}$. B. Bursting index (violin plot) and $p$-values $(p)$ of Wilcoxon-Mann-Whitney tests in Col-0 and fer-4 etiolated hypocotyls grown on a medium containing $1 \mathrm{nM}$ isoxaben with $0.7 \%$ or $2.5 \%$ agar. $\mathbf{C}$. Representative confocal images of Col-0 and fer-4 pavement cells, from seedlings grown on a medium containing $0.7 \%$ or $2.5 \%$ agar at $\mathrm{t}=4 \mathrm{DAG}$ and $\mathrm{t}=12 \mathrm{DAG}$ (propidium iodide staining). Scale $=100 \mu \mathrm{m}$. D. Bursting index (violin plot) and $p$-values $(p)$ of Wilcoxon-Mann-Whitney tests in Col-0 and fer-4 pavement cells, from seedlings grown on a medium containing $0.7 \%$ or $2.5 \%$ agar at $\mathrm{t}=4 \mathrm{DAG}$ and $\mathrm{t}=12$ DAG. E. Representative images of $\mathrm{Col}-0$ and $\mathrm{fer}-4$ cotyledons, grown on a medium containing $0.7 \%$ or $2.5 \%$ agar at $\mathrm{t}=4 \mathrm{DAG}, \mathrm{t}=8 \mathrm{DAG}$ and $\mathrm{t}=12 \mathrm{DAG}$. Scale $=1 \mathrm{~mm}$. F. Cotyledon area (violin plot) and $p$ values $(p)$ of Wilcoxon-Mann-Whitney tests in Col-0 and fer-4 seedlings, grown on a medium containing $0.35 \%, 0.7 \%, 1.25 \%$ or $2.5 \%$ agar at $\mathrm{t}=4 \mathrm{DAG}, \mathrm{t}=8 \mathrm{DAG}$ and $\mathrm{t}=12 \mathrm{DAG}$. 


\section{Cortical microtubule orient with predicted tensile stress after ablation in fer-4}

So far, all the tests suggest that FER is a major player in mechanosensing at the shoot, consistent with data obtained in the root (Shih 2014). However, it is not clear whether this involves the microtubule response to mechanical stress. To formally check this, we analysed the behaviour of cortical microtubules in response to local ablation in fer-4. From a mechanical standpoint, the sudden drop of turgor pressure in the ablated cells, together with epidermal tension (Kutschera and Niklas, 2007), generates a circumferential tensile stress around the dead cells (Hamant et al., 2008). Such a perturbation causes the cortical microtubules to reorient in the new maximal tensile stress direction (circumferential) around the ablation (Hamant et al., 2008)(Sampathkumar et al., 2014). We used the microtubule reporter $p$ PDF1::mCit-MBD to monitor the microtubule response in Col-0 and fer-4 cotyledons. As a $0.7 \%$ agar medium triggers widespread cell death in fer-4 (Figure 3C and Supplementary Figure 3A-D), all tests were performed on $2.5 \%$ agar. We measured both the anisotropy and the average orientation of cortical microtubule arrays (relative to the ablation site) in cells surrounding the ablation (Figure 4 and Supplementary Figure 4).

The anisotropy $(a)$ of cortical microtubule arrays was low in the WT immediately after ablation $\left(a_{C o l-0,0}=0.11 ; \mathrm{n}_{\mathrm{Col}-0, t 0}=248\right)$. It increased by $50 \%$ seven hours later, consistent with the coalignment of cortical microtubules with tensile stress $\left(a_{\text {Col- } 0, \mathrm{t} 7 \mathrm{~h}}=0.16 ; p<10^{-3} ; \mathrm{n}_{\text {Col- } 0, \mathrm{t} 7 \mathrm{~h}}=220\right.$; Figure 4E). In fer-4, cortical microtubule arrays were slightly less anisotropic (by 30\%) than the WT at $\mathrm{t}=0 \mathrm{~h}\left(a_{\text {fer }-4, \mathrm{t} 0}=0.073 ; p<10^{-3} ; \mathrm{n}_{\text {fer }-4, \mathrm{t} 0}=174\right)$. After seven hours, the anisotropy of cortical microtubule arrays increased by $129 \%$ and became comparable to that of the WT ( $a_{f e r-}$ $4, \mathrm{t} 7 \mathrm{~h}=0.17 ; p=0.26 ; n_{f e r-4, \mathrm{t} 7 \mathrm{~h}}=203$, Figure $\left.4 \mathrm{E}\right)$.

Immediately after the ablation, cortical microtubule arrays exhibited no preferred orientation (o) around the ablation, with an average angle of $47 \pm 25^{\circ}$ for the WT, which was not significantly different from that of fer-4 $\left(o_{\text {fer }-4, \mathrm{t} 0}=46 \pm 25^{\circ} ; p=0.69\right.$; Figure $4 \mathrm{~F}$ and $4 \mathrm{H}$, Supplementary Figure 4B). At t0, both distributions followed a uniform law on $0-90^{\circ}\left(p_{\text {Col- }}\right.$ $0, t_{0}=0.5 ; p_{f e r-4,0}=0.7 ;$ Kolmogorov-Smirnov test for uniformity). After seven hours, cortical microtubules did not follow a uniform distribution anymore $\left(p_{\text {Col- } 0, t 7 \mathrm{~h}}<10^{-3} ; p_{\text {fer }-4, \mathrm{t7h}}<10^{-3}\right.$; Kolmogorov-Smirnov test for uniformity) and became more circumferential around the ablation in both Col-0 and fer-4, with no significant difference between the genotypes (ocol-0,t7h $=28 \pm 23^{\circ}, o_{\text {fer }-4, \mathrm{th}}=31 \pm 25^{\circ} ; p=0.48$, Figure 4G and 4I, Supplementary Figure 4). 

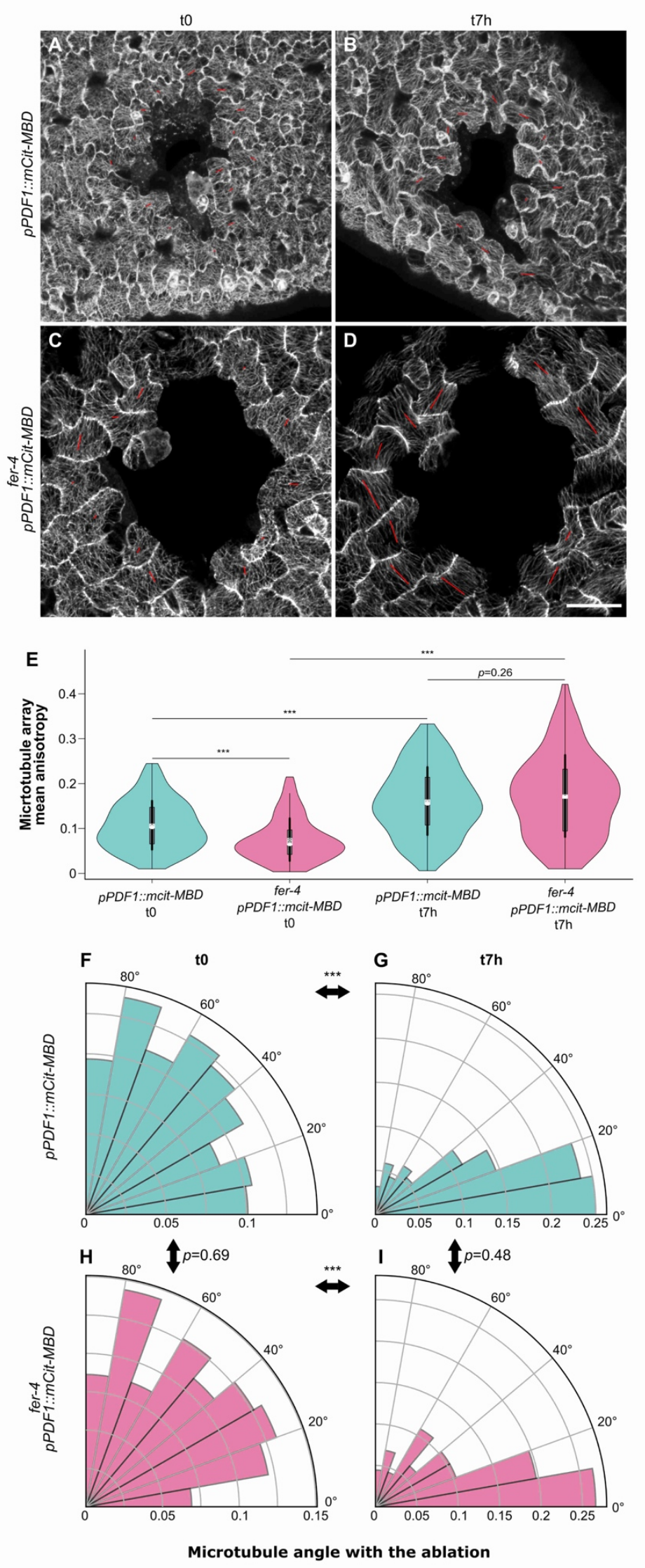

Figure 4. Cortical microtubule alignment with predicted maximal tensile stress in fer after ablation

All seedlings were grown on $2.5 \%$ agar.

A-D. Representative confocal images of $p P D F 1:: m C i t-M B D$ $(\mathrm{A}, \mathrm{B})$ and $f e r-4$ pPDF 1::mCit-MBD (C, D) pavement cells, immediately after an ablation (t0, A, C) and 7 hours later (t7h, B, D). The red bars indicate the average orientations of cortical microtubule arrays, and the length of the red bars is proportional to the anisotropy of the cortical microtubule arrays (using ImageJ FibrilTool). Scale $=50 \mu \mathrm{m}$.

E. Anisotropy (violin plot) of cortical microtubule arrays and $p$-values $(p)$ of WilcoxonMann-Whitney tests in cells surrounding the ablation site in pPDF1::mCit-MBD and fer-4 pPDF1::mCit-MBD pavement cells, immediately after an ablation (t0) and 7 hours later (t7h).

F-I. Cortical microtubule orientations (polar plots) and $p$-values $(p)$ of WilcoxonMann-Whitney tests in cells surrounding the ablation site in pPDF1::mCit-MBD (F, G) and fer-4 pPDF $1:: m$ Cit-MBD (H,I) pavement cells, immediately after an ablation (t0, F, H)) and seven hours later ( $\mathrm{t} 7 \mathrm{~h}, \mathrm{G}, \mathrm{I})$. 
Similar trends could be observed when using the $p 35 S:: G F P-T U B$ microtubule marker line: cortical microtubule orientation appeared circumferential around ablations in both WT and fer4 (Supplementary Figure 5). However, the diffuse fluorescent signal hindered quantitative analysis with FibrilTool. Because the microtubule response to ablation is comparable in fer-4 and in the WT, this formally shows that the microtubule response to stress can be independent from FER.

\section{FERONIA and microtubules independently contribute to the response to mechanical stress}

Our ablation results may seem at odds with the fact that fer-4 and bot 1-7 belong to the same cluster when analysing pavement cell circularity (see Figure 1D-E). We thus revisited our quantification of pavement cell shape to identify other shape descriptors amenable to discriminate bot 1-7 and fer-4. We focused on lobe size in pavement cells. In the katanin mutant bot1-7, which displayed a higher circularity than the WT (see Figure 1), the average basal lobe width was $3 \%$ smaller than that of the WT $\left(p<10^{-3} ; n_{\mathrm{WS}-4}=294 ; n_{\text {bot }-7}=252\right.$; Figure 5A). Although this is a rather small difference, this is consistent with a reduced microtubule response to stress in the katanin mutant. In contrast, the average basal lobe width in fer- 4 was $12 \%$ larger than that of the WT $\left(p<10^{-3} ; n_{\mathrm{Col}-0}=428 ; n_{f e r-4}=321\right.$; Figure $\left.5 \mathrm{~A}\right)$. Similar trends in basal lobe width were observed when seedlings were grown on $2.5 \%$ agar, albeit with lower values, also consistent with a reduced microtubule response to mechanical stress in such conditions (Supplementary Figure 6). Thus, both fer-4 and bot1-7 pavement cells exhibit higher circularity than the WT through different geometries. Pavement cell shape may thus reflect different responses to stress: reduced microtubule dynamics in bot1-7 would generate smaller lobes, whereas weaker walls in fer-4 would increase stress levels, leading to hyper-aligned cortical microtubules and larger lobes. Such hyper-aligned cortical microtubules can be observed in fer4 pavement cells when seedlings are grown on $0.7 \%$ agar (see Supplementary Figure 3C). Alternatively, and non-exclusively, the presence of dead cells in fer-4 may affect the stress pattern, and thus the cortical microtubule response, further increasing the circularity of pavement cells. 


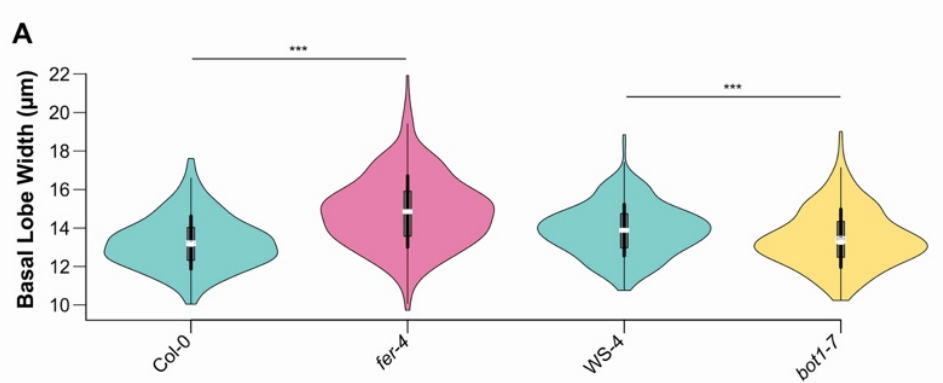

B

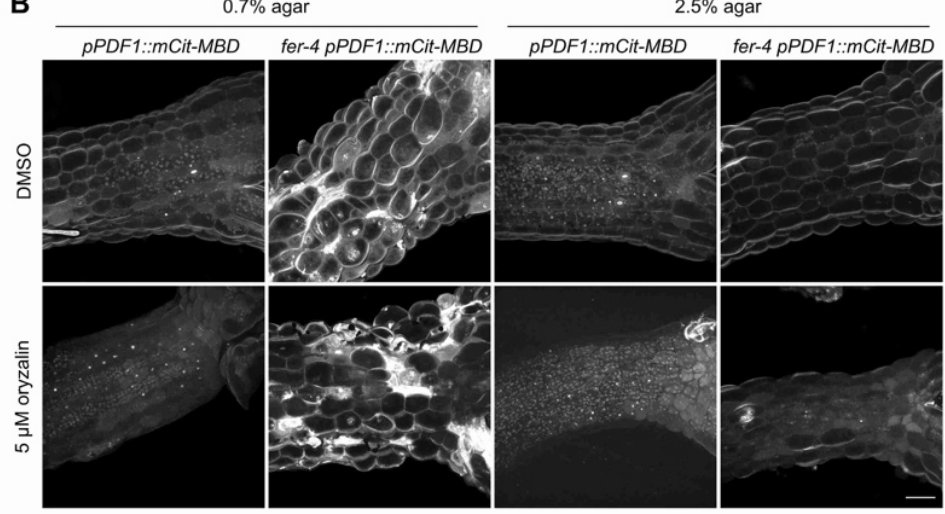

C

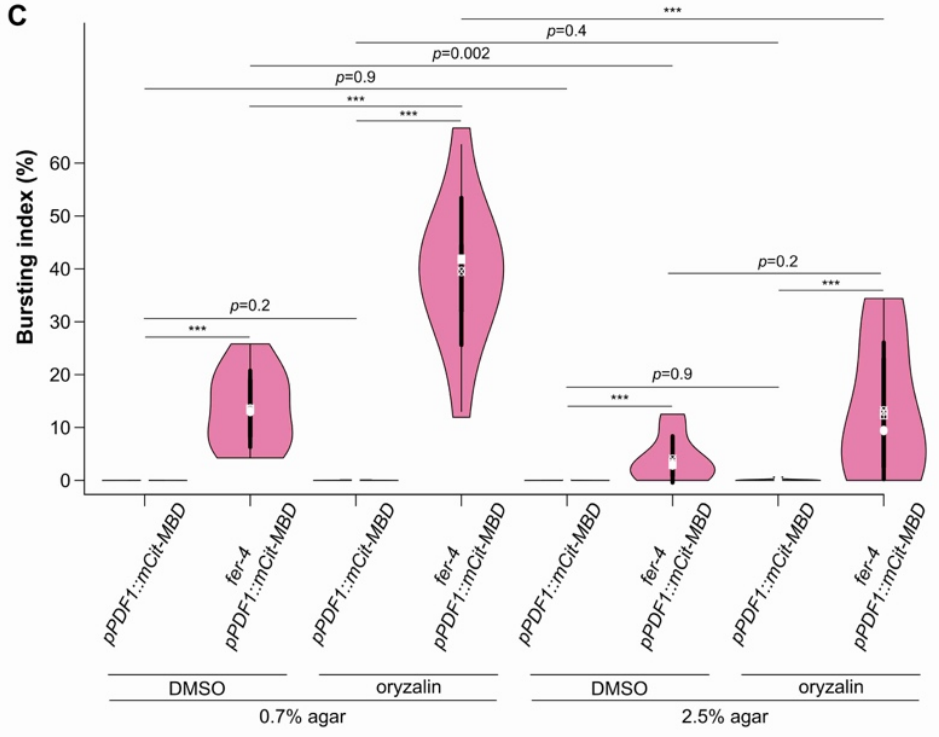

D
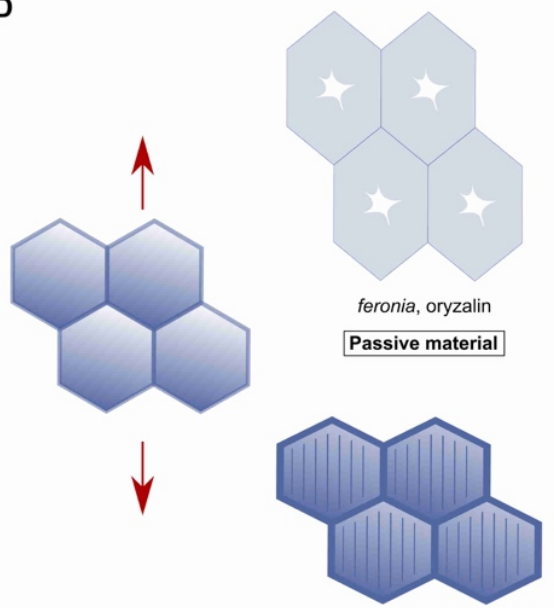

Wild type Active material
Cell wall deformation and failure

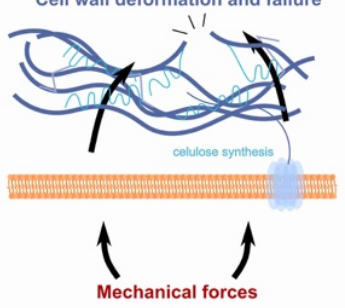

Cell wall reinforcement

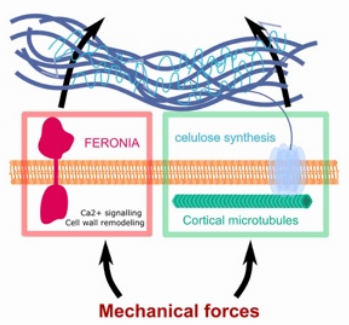

Figure 5. FER and

microtubules independently contribute to the response to mechanical stress

A. Basal lobe width (violin plot) of pavement cells and $p$ values $(p)$ of Dunn tests for the WT (Col-0, WS-4), katanin mutant (bot 1-7), and fer-4.

Seedlings were grown on $0.8 \%$ agar.

B. Representative confocal images of $p P D F 1:: m C i t-M B D$ and fer-4 pPDF1::mCit$M B D$ hypocotyls grown on $0.7 \%$ and $2.5 \%$ agar with and without $5 \mu \mathrm{m}$ of oryzalin. Scale bar $=50 \mu \mathrm{m}$.

C. Bursting index (violin plot) and $p$-values $(p)$ of WilcoxonMann-Whitney tests in pPDF1::mCit-MBD and fer-4 pPDF1::mCit-

$M B D$ hypocotyls grown on $0.7 \%$ or $2.5 \%$ agar, with and without $5 \mu \mathrm{m}$ of oryzalin.

D. In the WT (bottom), cells resist mechanical stress (red arrows) by two independent pathways (microtubuledependent cellulose synthesis and FER-dependent wall reinforcement). In absence of both FER and microtubules (top), cells deform like passive materials and ultimately burst. 
Treatment with the microtubule depolymerizing drug oryzalin has previously been shown to induce cell bursting events in the largest cells at the shoot apical meristem (Sapala et al., 2018), mimicking the bursting cell phenotype we observe in hypocotyls and cotyledons in fer-4. If both pathways are truly independent, then one should expect additive behaviors. To test this prediction, we observed hypocotyl growth when both pathways are down, by applying oryzalin on fer-4 mutants. On $0.7 \%$ agar, bursting cells appeared in both oryzalin-treated hypocotyls and control ones in fer- 4 . The bursting index increased by $80 \%$ in oryzalin-treated fer- 4 hypocotyls, consistent with an additive role of both pathways in wall integrity $\left(p_{f e r-4,0.7 \% a g a r}=0.001 ; \mathrm{n}_{f e r}\right.$ 4,DMSO, $0.7 \%$ agar $=10 ; \mathrm{n}_{\text {fer- }-4 \text {,oryzalin, } 0.7 \% \mathrm{agar}}=11$, Figure $5 \mathrm{~B}-\mathrm{C}$ ). To relate this phenotype to stress levels, we performed the same experiment on $2.5 \%$ agar, and found a reduction in the bursting cells in all conditions (by $87 \%$ on DMSO; $p_{f e r-4, \mathrm{DMSO}}=0.002 ; \mathrm{n}_{\text {fer }-4, \mathrm{DMSO}, 0.7 \% \mathrm{agar}}=10 ; \mathrm{n}_{\text {fer }-4, \mathrm{DMSO}, 2.5 \% \mathrm{agar}}=9$;

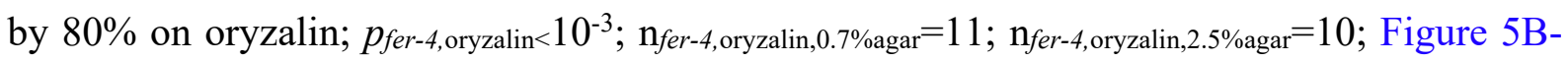
C). This further confirms that FERONIA and microtubules independently contribute to the response to stress.

\section{Discussion}

From a reverse genetic screen, we show that FERONIA plays a primary role in the cell response to mechanical stress in the shoot. We also find that the microtubule response to stress does not depend on FER. This provides a scenario in which the mechanical feedback in the shoot involves two independent modules, cortical microtubules and FER (Figure 5D).

This result is consistent with recent findings showing how pectin and cellulose deposition are controlled with largely disconnected networks: Pectins are important for the initiation of pavement cell formation, and the deposition of cellulose microfibril rather amplifies a preexisting mechanical pattern (Bidhendi et al., 2019). Similarly, mechanical polarities in growing hypocotyl cells precedes the cellulose-derived mechanical anisotropy in hypocotyl cells (Peaucelle et al., 2015).

We also provide evidence that the fer mutant can be largely rescued though changes in the mechanical environment of the plant despite its central and somewhat pleiotropic role in plant development. This echoes recent findings where essential regulators are found optional, when challenged with different mechanical environments. This is the case for instance for key regulators of pectin synthesis, where decreasing tensile stress can rescue cell-cell adhesion defects in the quasimodo mutants (Verger et al., 2018). This was also shown for the katanin 
mutant, where increasing tensile stress levels with isoxaben treatment can generate WT-like cortical microtubule arrays (Uyttewaal et al., 2012). More recently, this was even extended to signaling in the context of apical hook formation: arf7 arf19 auxin transduction mutant exhibit a WT phenotype when mechanically constrained (Baral et al., 2020).

If the microtubule response to stress does not depend on FER, what could be the most relevant mechanotransduction pathway? As shown with optical tweezers, pulling on microtubules promote their polymerization in vitro (Franck et al., 2007)(Trushko et al., 2013). Cortical microtubules also align with predicted maximal tension when protoplasts are confined in rectangular microwells and pressurized by hypo-osmotic conditions (Colin et al., 2020). These data, together with the fer-independent microtubule response to stress, further support the hypothesis that microtubules may be their own mechanosensor (Hamant et al., 2019).

Conversely, the final microtubule (and cellulose microfibril) alignment likely results from a combination of several cues, beyond tensile stress. Cell geometry can affect microtubule behaviour, independent of cortical tension. In particular, due to their high persistence length, microtubules tend to become longitudinal in vitro (Cosentino Lagomarsino et al., 2007) or in depressurized protoplasts (Durand-Smet et al., 2020) (Colin et al., 2020). Furthermore, cell edge factors can affect cortical microtubule behaviour, leading to cell-scale aligned arrays (Ambrose et al., 2011)(Chakrabortty et al., 2018)(Kirchhelle et al., 2019). Although microtubule, FER, stress and cell geometry can be uncoupled in experiments, their interplay may provide synergies in vivo. In particular, the deposition of matrix material in the wall depends on exocytosis, which is also promoted by membrane tension. Conversely, affecting matrix deposition may weaken the wall and increase the tensile stress levels. Consistently, microtubule arrays exhibit an enhanced anisotropy when the cell edge GTPase Rab-A5c dependent trafficking is affected in roots (Kirchhelle et al., 2019).

All living organisms constantly sense and respond to mechanical stress. By impairing two major mechanosensing players, we show what plants looks like when cells become unable to respond to stress. We find that cells switch to a more passive mode, like a balloon or a soap bubble: cell behaviour scales with stress properties, and usually they die ultimately. Defining living systems as active material is thus particularly suited to understand how cells and tissue behave in response to fluctuations in their physical environment. The physics of active matter, and the associated mechano-chemical couplings, may be essential to revisit the role of many plant signaling pathways too. 


\section{Material and Methods}

\section{Plant material and growth conditions}

All plants were in the Columbia-0 (Col-0) ecotype, except the bot1-7 mutant, which was in the Wassilewskija (WS-4) ecotype (Supplementary Table 1).

Seeds were surface sterilized and individually sown on Murashige and Skoog medium (MS medium, Duchefa, Haarlem, the Netherlands) or Arabidopsis medium (custom-made Duchefa 'Arabidopsis' medium (DU0742.0025, Duchefa Biochemie) with different agar concentrations (see Supplementary Table 2 for detailed description of the different medium used). For drug treatments, mediums were supplemented with isoxaben (Sigma) or oryzalin (Chem Service Inc.) from stock solutions in dimethyl sulfoxide (DMSO, Sigma). All plants were placed 2 days in the dark at $4^{\circ} \mathrm{C}$ then transferred in a $20^{\circ} \mathrm{C}$ long-days growth chamber. When seedlings were maintained in the dark, petri dishes were covered with aluminium foil in the $20^{\circ} \mathrm{C}$ long-days growth chamber.

\section{Image acquisition}

Samples were imaged with either a SP8 confocal microscope (Leica Microsystems) equipped with a 25x long-distance water objective $(\mathrm{NA}=0.95)$, an Epson Perfection 2400 scanner, or a Leica MZ12 microscope (As specified in Supplementary Table 2). Samples were stained for 10 minutes with a propidium iodide solution (Sigma; PI stains wall pectins and thus marks cell contours). Ablations (Figure 4) were performed manually with a fine needle (Minutien pin, 0.15 $\mathrm{mm}$ rod diameter, $0.02 \mathrm{~mm}$ tip width, RS-6083-15, Roboz Surgical Instrument Co.) as described in (Verger et al., 2018). In all confocal microscopy images, $0.5 \mu \mathrm{m}$ thick optical slices were acquired.

For every experiment, three biological replicates or more were obtained. Col-0 or WS-4 seedlings were included as controls in all experiments and replicates. Note that all of the Col-0 images used in the first figure of this study have also been used in (Erguvan et al., 2019), as templates to introduce the SurfCut ImageJ tool (see below in Image analysis) for cell contour extraction. 


\section{Image analysis}

Pavement cell shape were obtained by first processing confocal images with MorphoGraphX (http://www.mpipz.mpg.de/MorphoGraphX) (Barbier de Reuille et al., 2015) to obtain cell contours in a $2.5 \mathrm{D}$ epidermal surface (Figures 1 and $5 \mathrm{~A}$ ) or SurfCut (https://github.com/sverger/SurfCut) (Erguvan et al., 2019) to extract the flattened cell contours (Figure 3E). The cell contour images were then processed with PaCeQuant (Möller et al., 2017), an ImageJ plugin quantifying up to 27 shape descriptors of pavement cells. Hypocotyl length (Figure 2) were measured manually with ImageJ (https://fiji.sc/). Cell burst area (Figure 3 and $5 \mathrm{C}-\mathrm{D})$ was measured manually with Image J after extracting the flattened cell contours with SurfCut (https://github.com/sverger/SurfCut) (Erguvan et al., 2019). Cotyledon area (Figure 3) was measured manually with ImageJ. Microtubule organization (Figure 4) was quantified with FibrilTool (Boudaoud et al., 2014) after flattening the images with SurfCut and denoising them (ROF Denoise, Theta=25) in ImageJ, as performed in (Verger et al., 2018). Ablations (Figure 4) were performed manually with a fine needle (Minutien pin, $0.15 \mathrm{~mm}$ rod diameter, $0.02 \mathrm{~mm}$ tip width, RS-6083-15, Roboz Surgical Instrument Co.) as described in (Verger et al., 2018). After image analyses, the luminosity and contrast of all images presented in this study were enhanced to help visualization.

\section{Statistical analysis}

Statistical analyses were performed with R software (https://www.R-project.org). The sample size is indicated in the main text and in the figure legends. For pavement cell shapes (Figures 1, 5A and 6A), we used PaCeQuantAna, the R script that accompanies the PaCeQuant analysis (Möller et al., 2017), and the FactoMineR and factoextra R libraries for the PCA (Lê et al., 2008). Violin plots were shown with the corresponding $p$-value of Kruskal-Wallis tests. For hypocotyl length (Figure 2), the control distribution of hypocotyl length was standardized and the same parameters $\left(\mu_{\mathrm{ctrl}}, \mathrm{o}_{\mathrm{ctrl}}\right)$ used to shift the isoxaben distribution similarly (Sup. Figure 2). A Wilcoxon-Mann-Whitney test was then performed on the shifted distributions of the mutant and of the WT for each genotype. For the orientation of microtubules, a Kolmogorov-Smirnov test was performed to compare the angle distributions to a uniform distribution between 0 and $90^{\circ}$. All other quantitative measures were compared using Wilcoxon-Mann-Whitney tests. As a Wilcoxon-Mann-Whitney test can be directed or not, the p-value shown in all experiments with a Wilcoxon-Mann-Whitney test was that of a non-directed test for non-significant p-value (to ensure that neither distribution was higher than the other) and that of a directed test in the 
significant direction for a significant p-value. Differences with $p$-values that were under $1 \%$ were considered significant, and those between the commonly used thresholds of $5 \%$ and $1 \%$ were considered as tendencies. All \pm values referred to the standard deviation of the distribution.

\section{Acknowledgments}

We are thankful to our colleagues at the plant reproduction and development lab for their comments and feedback on this manuscript. We thank Platim for help with imaging. This work was supported by the European Research Council (ERC-2013-CoG-615739 'MechanoDevo'.

\section{References}

Ambrose, C., Allard, J. F., Cytrynbaum, E. N. and Wasteneys, G. O. (2011). A CLASPmodulated cell edge barrier mechanism drives cell-wide cortical microtubule organization in Arabidopsis. Nat. Commun. 2, 430.

Anderson, C. T. and Kieber, J. J. (2020). Dynamic Construction, Perception, and Remodeling of Plant Cell Walls. Annu. Rev. Plant Biol. 71, 39-69.

Bacete, L. and Hamann, T. (2020). The Role of Mechanoperception in Plant Cell Wall Integrity Maintenance. Plants 9, 574.

Baral, A., Morris, E., Aryal, B., Jonsson, K., Verger, S., Xu, T., Bennett, M., Hamant, O. and Bhalerao, R. P. (2020). External mechanical cues reveal core molecular pathway behind tissue bending in plants. Plant Biology.

Barbier de Reuille, P., Routier-Kierzkowska, A.-L., Kierzkowski, D., Bassel, G. W., Schüpbach, T., Tauriello, G., Bajpai, N., Strauss, S., Weber, A., Kiss, A., et al. (2015). MorphoGraphX: A platform for quantifying morphogenesis in 4D. eLife 4, 05864.

Bidhendi, A. J., Altartouri, B., Gosselin, F. P. and Geitmann, A. (2019). Mechanical Stress Initiates and Sustains the Morphogenesis of Wavy Leaf Epidermal Cells. Cell Rep. 28, 12371250.e6.

Boudaoud, A., Burian, A., Borowska-Wykręt, D., Uyttewaal, M., Wrzalik, R., Kwiatkowska, D. and Hamant, O. (2014). FibrilTool, an ImageJ plug-in to quantify fibrillar structures in raw microscopy images. Nat. Protoc. 9, 457-463.

Chakrabortty, B., Willemsen, V., de Zeeuw, T., Liao, C.-Y., Weijers, D., Mulder, B. and Scheres, B. (2018). A Plausible Microtubule-Based Mechanism for Cell Division Orientation in Plant Embryogenesis. Curr. Biol. 28, 3031-3043.e2.

Colin, L., Chevallier, A., Tsugawa, S., Gacon, F., Godin, C., Viasnoff, V., Saunders, T. E. and Hamant, O. (2020). Cortical tension overrides geometrical cues to orient microtubules in 
confined protoplasts. Proc. Natl. Acad. Sci. U. S. A. 117, 32731-32738.

Cosentino Lagomarsino, M., Tanase, C., Vos, J. W., Emons, A. M. C., Mulder, B. M. and Dogterom, M. (2007). Microtubule organization in three-dimensional confined geometries: evaluating the role of elasticity through a combined in vitro and modeling approach. Biophys. J. 92, 1046-1057.

Cosgrove, D. J. (2005). Growth of the plant cell wall. Nat. Rev. Mol. Cell Biol. 6, 850-861.

Durand-Smet, P., Spelman, T. A., Meyerowitz, E. M. and Jönsson, H. (2020). Cytoskeletal organization in isolated plant cells under geometry control. Proc. Natl. Acad. Sci. 117, 1739917408.

Engelsdorf, T., Gigli-Bisceglia, N., Veerabagu, M., McKenna, J. F., Vaahtera, L., Augstein, F., Van der Does, D., Zipfel, C. and Hamann, T. (2018). The plant cell wall integrity maintenance and immune signaling systems cooperate to control stress responses in Arabidopsis thaliana. Sci. Signal. 11, eaao3070.

Engelsdorf, T., Kjaer, L., Gigli-Bisceglia, N., Vaahtera, L., Bauer, S., Miedes, E., Wormit, A., James, L., Chairam, I., Molina, A., et al. (2019). Functional characterization of genes mediating cell wall metabolism and responses to plant cell wall integrity impairment. $B M C$ Plant Biol. 19, 320.

Erguvan, Ö., Louveaux, M., Hamant, O. and Verger, S. (2019). ImageJ SurfCut: a userfriendly pipeline for high-throughput extraction of cell contours from 3D image stacks. $B M C$ Biol. 17, 38 .

Fan, Y., Burkart, G. M. and Dixit, R. (2018). The Arabidopsis SPIRAL2 Protein Targets and Stabilizes Microtubule Minus Ends. Curr. Biol. CB 28, 987-994.e3.

Feng, W., Kita, D., Peaucelle, A., Cartwright, H. N., Doan, V., Duan, Q., Liu, M.-C., Maman, J., Steinhorst, L., Schmitz-Thom, I., et al. (2018). The FERONIA Receptor Kinase Maintains Cell-Wall Integrity during Salt Stress through Ca2+ Signaling. Curr. Biol. CB 28, 666-675.e5.

Franck, A. D., Powers, A. F., Gestaut, D. R., Gonen, T., Davis, T. N. and Asbury, C. L. (2007). Tension applied through the Dam1 complex promotes microtubule elongation providing a direct mechanism for length control in mitosis. Nat. Cell Biol. 9, 832-837.

Fu, Y., Gu, Y., Zheng, Z., Wasteneys, G. and Yang, Z. (2005). Arabidopsis interdigitating cell growth requires two antagonistic pathways with opposing action on cell morphogenesis. Cell 120, 687-700.

Gonneau, M., Desprez, T., Martin, M., Doblas, V. G., Bacete, L., Miart, F., Sormani, R., Hématy, K., Renou, J., Landrein, B., et al. (2018). Receptor Kinase THESEUS1 Is a Rapid Alkalinization Factor 34 Receptor in Arabidopsis. Curr. Biol. CB 28, 2452-2458.e4.

Green, P. and King, A. (1966). A mechanism for the origin of specifically oriented textures in development with special reference to Nitella wall texture. Aust J Biol Sci 421-437. 
Hamant, O. and Saunders, T. E. (2020). Shaping Organs: Shared Structural Principles Across Kingdoms. Annu. Rev. Cell Dev. Biol. 36, 385-410.

Hamant, O., Heisler, M. G., Jonsson, H., Krupinski, P., Uyttewaal, M., Bokov, P., Corson, F., Sahlin, P., Boudaoud, A., Meyerowitz, E. M., et al. (2008). Developmental patterning by mechanical signals in Arabidopsis. Science 322, 1650-1655.

Hamant, O., Inoue, D., Bouchez, D., Dumais, J. and Mjolsness, E. (2019). Are microtubules tension sensors? Nat. Commun. 10, 2360.

Heisler, M. G., Hamant, O., Krupinski, P., Uyttewaal, M., Ohno, C., Jönsson, H., Traas, J. and Meyerowitz, E. M. (2010). Alignment between PIN1 Polarity and Microtubule Orientation in the Shoot Apical Meristem Reveals a Tight Coupling between Morphogenesis and Auxin Transport. PLoS Biol. 8,.

Hejnowicz, Z., Rusin, A. and Rusin, T. (2000). Tensile Tissue Stress Affects the Orientation of Cortical Microtubules in the Epidermis of Sunflower Hypocotyl. J. Plant Growth Regul. 19, $31-44$.

Hématy, K., Sado, P.-E., Van Tuinen, A., Rochange, S., Desnos, T., Balzergue, S., Pelletier, S., Renou, J.-P. and Höfte, H. (2007). A receptor-like kinase mediates the response of Arabidopsis cells to the inhibition of cellulose synthesis. Curr. Biol. CB 17, 922-931.

Hervieux, N., Dumond, M., Sapala, A., Routier-Kierzkowska, A.-L., Kierzkowski, D., Roeder, A. H. K., Smith, R. S., Boudaoud, A. and Hamant, O. (2016). A Mechanical Feedback Restricts Sepal Growth and Shape in Arabidopsis. Curr. Biol. CB.

Ishida, T., Kaneko, Y., Iwano, M. and Hashimoto, T. (2007). Helical microtubule arrays in a collection of twisting tubulin mutants of Arabidopsis thaliana. Proc. Natl. Acad. Sci. 104, 8544-8549.

Kirchhelle, C., Garcia-Gonzalez, D., Irani, N. G., Jérusalem, A. and Moore, I. (2019). Two mechanisms regulate directional cell growth in Arabidopsis lateral roots. eLife 8, e47988.

Kutschera, U. and Niklas, K. J. (2007). The epidermal-growth-control theory of stem elongation: an old and a new perspective. J. Plant Physiol. 164, 1395-1409.

Lê, S., Josse, J. and Husson, F. (2008). FactoMineR: An R Package for Multivariate Analysis. J. Stat. Softw. 25, 1-18.

Lin, W., Tang, W., Anderson, C. T. and Yang, Z. (2018). FERONIA's sensing of cell wall pectin activates ROP GTPase signaling in Arabidopsis. Plant Biology.

Majda, M., Grones, P., Sintorn, I.-M., Vain, T., Milani, P., Krupinski, P., ZagórskaMarek, B., Viotti, C., Jönsson, H., Mellerowicz, E. J., et al. (2017). Mechanochemical Polarization of Contiguous Cell Walls Shapes Plant Pavement Cells. Dev. Cell 43, 290-304.e4.

Manfield, I. W., Orfila, C., McCartney, L., Harholt, J., Bernal, A. J., Scheller, H. V., Gilmartin, P. M., Mikkelsen, J. D., Paul Knox, J. and Willats, W. G. T. (2004). Novel cell wall architecture of isoxaben-habituated Arabidopsis suspension-cultured cells: global 
transcript profiling and cellular analysis. Plant J. Cell Mol. Biol. 40, 260-275.

Matsumoto, S., Kumasaki, S., Soga, K., Wakabayashi, K., Hashimoto, T. and Hoson, T. (2010). Gravity-induced modifications to development in hypocotyls of Arabidopsis tubulin mutants. Plant Physiol. 152, 918-926.

Möller, B., Poeschl, Y., Plötner, R. and Bürstenbinder, K. (2017). PaCeQuant: A Tool for High-Throughput Quantification of Pavement Cell Shape Characteristics. Plant Physiol. 175, 998-1017.

Nakamura, M., Lindeboom, J. J., Saltini, M., Mulder, B. M. and Ehrhardt, D. W. (2018). SPR2 protects minus ends to promote severing and reorientation of plant cortical microtubule arrays. J. Cell Biol. 217, 915-927.

Paredez, A. R., Somerville, C. R. and Ehrhardt, D. W. (2006). Visualization of cellulose synthase demonstrates functional association with microtubules. Science 312, 1491-1495.

Peaucelle, A., Wightman, R. and Höfte, H. (2015). The Control of Growth Symmetry Breaking in the Arabidopsis Hypocotyl. Curr. Biol. CB 25, 1746-1752.

Sampathkumar, A., Krupinski, P., Wightman, R., Milani, P., Berquand, A., Boudaoud, A., Hamant, O., Jönsson, H. and Meyerowitz, E. M. (2014). Subcellular and supracellular mechanical stress prescribes cytoskeleton behavior in Arabidopsis cotyledon pavement cells. eLife 3, e01967.

Sapala, A., Runions, A., Routier-Kierzkowska, A.-L., Das Gupta, M., Hong, L., Hofhuis, H., Verger, S., Mosca, G., Li, C.-B., Hay, A., et al. (2018). Why plants make puzzle cells, and how their shape emerges. eLife 7 ,.

Scheible, W. R., Eshed, R., Richmond, T., Delmer, D. and Somerville, C. (2001). Modifications of cellulose synthase confer resistance to isoxaben and thiazolidinone herbicides in Arabidopsis Ixr1 mutants. Proc. Natl. Acad. Sci. U. S. A. 98, 10079-10084.

Schopfer, P. (2006). Biomechanics of plant growth. Am. J. Bot. 93, 1415-1425.

Shih, H.-W., Miller, N. D., Dai, C., Spalding, E. P. and Monshausen, G. B. (2014). The receptor-like kinase FERONIA is required for mechanical signal transduction in Arabidopsis seedlings. Curr. Biol. CB 24, 1887-1892.

Takatani, S., Verger, S., Okamoto, T., Takahashi, T., Hamant, O. and Motose, H. (2020). Microtubule Response to Tensile Stress Is Curbed by NEK6 to Buffer Growth Variation in the Arabidopsis Hypocotyl. Curr. Biol. S0960982220301974.

Trinh, D.-C., Alonso-Serra, J., Asaoka, M., Colin, L., Cortes, M., Malivert, A., Takatani, S., Zhao, F., Traas, J., Trehin, C., et al. (2021). How Mechanical Forces Shape Plant Organs. Curr. Biol. 31, R143-R159.

Trushko, A., Schäffer, E. and Howard, J. (2013). The growth speed of microtubules with XMAP215-coated beads coupled to their ends is increased by tensile force. Proc. Natl. Acad. Sci. U. S. A. 110, 14670-14675. 
Uyttewaal, M., Burian, A., Alim, K., Landrein, B., Borowska-Wykret, D., Dedieu, A., Peaucelle, A., Ludynia, M., Traas, J., Boudaoud, A., et al. (2012). Mechanical stress acts via katanin to amplify differences in growth rate between adjacent cells in Arabidopsis. Cell 149, $439-451$.

Verger, S., Long, Y., Boudaoud, A. and Hamant, O. (2018). A tension-adhesion feedback loop in plant epidermis. eLife $\mathbf{7}$,.

Vőfély, R. V., Gallagher, J., Pisano, G. D., Bartlett, M. and Braybrook, S. A. (2019). Of puzzles and pavements: a quantitative exploration of leaf epidermal cell shape. New Phytol. 221, 540-552.

Wightman, R., Chomicki, G., Kumar, M., Carr, P. and Turner, S. R. (2013). SPIRAL2 determines plant microtubule organization by modulating microtubule severing. Curr. Biol. $C B$ 23, 1902-1907.

Williamson, R. (1990). Alignment of cortical microtubules by anisotropic wall stresses. Aust J Plant Physiol 601-613.

Wolfenson, H., Yang, B. and Sheetz, M. P. (2019). Steps in Mechanotransduction Pathways that Control Cell Morphology. Annu. Rev. Physiol. 81, 585-605.

Zhang, C., Halsey, L. E. and Szymanski, D. B. (2011). The development and geometry of shape change in Arabidopsis thalianacotyledon pavement cells. BMC Plant Biol. 11, 27. 